\title{
Plasma HER2ECD a promising test for patient prognosis and prediction of response in HER2 positive breast cancer: results of a randomized study - SAKK 22/99
}

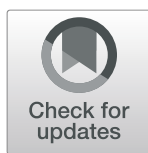

Serenella Eppenberger-Castori ${ }^{*}$ (D), Dirk Klingbie ${ }^{2,3}$, Thomas Ruhstaller $^{4,5}$, Daniel Dietrich ${ }^{2}$, Daniel Alexander Rufle ${ }^{1}$, Karin Rothgiesser ${ }^{2}$, Olivia Pagani ${ }^{6 \dagger}$ and Beat Thürlimann ${ }^{7,5+}$

\begin{abstract}
Background: The HER2 extracellular domain shed in blood (HER2 $2_{E C D}$ ) is reported to rise and fall in parallel with HER2+ breast cancer behavior. In this study, we evaluated the clinical relevance of plasma HER2ECD values in patients with metastatic breast cancer treated in the SAKK22/99 trial comparing trastuzumab monotherapy followed by trastuzumab-chemotherapy combination at progression versus upfront combination therapy.

Methods: Quantitative assessment of plasma HER2ECD was performed in 133 patients at baseline; after 2-24 h; at 3 weeks; at first response evaluation (8-9 weeks); and at tumor progression. Associations with tumor characteristics, disease course and trial treatment were evaluated.

Results: Baseline HER2ECD levels were stable within $24 \mathrm{~h}$ after the first trastuzumab injection. These plasma values correlated positively with the HER2 gene ratio $\left(r_{s}=0.39, P<0.001\right)$ and HER2 protein expression levels $\left(r_{s}=0.36\right.$, $P<0.001)$ but not with ER/PR status of the primary tumor. HER2 $E_{E C D}$ baseline levels were positively associated with the presence of visceral disease $(P=0.05)$ and poor patients' outcome (Cox-regression: $P=0.009)$. Patients with high baseline levels $(\geq 35 \mathrm{ng} / \mathrm{ml})$ had the worst overall survival $(P=0.03)$ if treated with upfront combination therapy. Conversely, patients with low HER2ECD baseline values $(<15 \mathrm{ng} / \mathrm{ml})$ had longer time to progression on combined trastuzumab-chemotherapy when first treated with trastuzumab monotherapy $(P=0.02)$. Monitoring HER2ECD levels during the course of the trial revealed significant time $(P=0.001)$ and time-treatment arm interactions $(P=0.0007)$. Under upfront trastuzumab alone, the HER2 ECD levels remained stable until just before disease progression. In patients responding to combination treatment HER2 ECD levels decreased to $\geq 20 \%$.

Conclusions: Plasma HER2ECD levels in patients with metastatic breast cancer reflect HER2 disease status. This robust biomarker might help identifying patients without visceral disease profiting from a sequential treatment's modality. Monitoring HER2 ECD levels during trastuzumab monotherapy could help defining the optimal time to introduce chemotherapy.
\end{abstract}

Trial registration: Registration Number by ClinicalTrials.gov: NCT00004935, Trial number: SAKK22/99. Registered on 27 January 2003.

Keywords: HER2+ breast cancer, Plasma HER2ECD levels, Baseline, Trastuzumab, Sequential therapy, Upfront combined therapy

\footnotetext{
* Correspondence: sere.eppenberger@unibas.ch

${ }^{\dagger}$ Olivia Pagani and Beat Thürlimann contributed equally to this work.

'University Hospital Basel, Institute of Human Genetic and Pathology,

Schönbeinstrasse 40, CH-4031 Basel, Switzerland

Full list of author information is available at the end of the article
}

(c) The Author(s). 2020 Open Access This article is distributed under the terms of the Creative Commons Attribution 4.0 International License (http://creativecommons.org/licenses/by/4.0/), which permits unrestricted use, distribution, and reproduction in any medium, provided you give appropriate credit to the original author(s) and the source, provide a link to the Creative Commons license, and indicate if changes were made. The Creative Commons Public Domain Dedication waiver (http://creativecommons.org/publicdomain/zero/1.0/) applies to the data made available in this article, unless otherwise stated. 


\section{Background}

Epidermal growth factor receptor-2 (HER2) is an oncogene of key importance in breast cancer (BC). Its amplification occurs in $15-25 \%$ of primary BC patients and is age-dependent $[1,2]$. HER2 amplification identifies an intrinsic subtype [3] of particularly aggressive $\mathrm{BC}$ correlating with higher levels of proteases [4]. HER2 represents the target of monoclonal antibodies, such as trastuzumab $(\mathrm{T})$, pertuzumab and $T-D M 1$, effective in the cure of patients with HER2 positive $\mathrm{BC}[5,6]$ as well as of tyrosine kinase inhibitors (TKIs) $[7,8]$. This protein located in the cytosolic membrane is composed of three domains: the internal tyrosine kinase reactive part, the transmembrane lipophilic linker and the extracellular carboxyl-terminal tail called extracellular domain (ECD) [9]. Metalloproteases can cleave the ECD from the cell surface. The HER $2_{\mathrm{ECD}}$ with a length of p105 represents the shed ECD product, which is circulating in blood of patients with $\mathrm{BC}$ cells overexpressing this oncogene and high levels of proteases. An enzyme-linked immunosorbent assay has been developed to dose HER $2_{\mathrm{ECD}}$ blood levels and proposed as peripheral marker for monitoring disease progression and predict therapy response [10-12]. This is of clinical interest since treatment of advanced BC is historically based on the HER2 status of the primary tumor and several studies have suggested a possible clinically significant discordance of up to $42 \%$ between the HER2 status at primary and later disease stages $[13,14]$. The discrepant HER2 status during the course of the disease is however reported to be minimal (2\%) when based on in situ hybridization detections and performed with exactly the same methods in a single center [15].

The HER $2_{\mathrm{ECD}}$ is detectable in serum and plasma, but the majority of studies analyze serum samples. The HER $2_{\text {ECD }}$ test is Food \& Drug Administration (FDA) approved and numerous clinical studies have demonstrated that both a HER $2_{\text {ECD }}$ serum level $>15 \mathrm{ng} / \mathrm{ml}$ and a reduction of $\geq 20 \%$ between 2 successive blood draws is predictive for significant trastuzumab response [16].

The present study was designed as translational research investigation of the SAKK22/99 clinical study [17] to investigate associations of plasma baseline HER $2_{\text {ECD }}$ values and longitudinal time points' variations with treatment efficacy to possibly improve identification of patients requiring upfront combination of trastuzumab with chemotherapy (TChemo).

\section{Methods}

\section{Inclusion criteria for the translational sub-protocol and} ethical considerations

In short, women with histologically proven HER2-positive advanced $\mathrm{BC}$ without previous trastuzumab treatment or brain/meningeal involvement or concomitant serious diseases were randomly assigned (1:1) to trastuzumab alone followed, at progressive disease (PD), by combination with chemotherapy (Arm A) versus the upfront TChemo (Arm B).

The primary endpoint of this superiority trial was time to progression (TTP) on combined trastuzumabchemotherapy (TTP-TChemo) in both arms. Secondary endpoints included response rate, TTP, overall survival, quality of life and toxicity. The T loading dose of $4 \mathrm{mg} /$ $\mathrm{kg}$ intravenous (iv) was followed by $2 \mathrm{mg} / \mathrm{kg}$ iv weekly. In the initial 1st-line population $(n=84)$, chemotherapy was weekly paclitaxel $90 \mathrm{mg} / \mathrm{m} 2$ iv (3/4 weeks). After the amendment, chemotherapy was at the investigator's choice (taxanes, vinorelbine, cisplatin) according to label indications/schedules. Chemotherapy could be stopped after $\geq 6$ cycles in responding patients or after unacceptable toxicity, trastuzumab was continued until progression.

We collected and immediately centrifuged EDTA blood samples at baseline before the first trastuzumab infusion and 2-24 h afterwards and before chemotherapy in case of Arm B. Further plasma sampling occurred after 3 weeks, at first response evaluation (8-9 weeks), at tumor progression, and whenever feasible at later clinical assessments. The centers shipped plasma samples overnight at room temperature to a central laboratory in Basel. All patients signed a specific informed consent. Two patients withdrew their consent during the course of the investigation and we appropriately destroyed the correspondent plasma samples. Baseline HER2ECD values were obtained from 66 and 67 patients out of 87 and 88 patients enrolled in Arm A and B, respectively. Details of the clinical pathological issues of this subset of patients are summarized in Table 1.

\section{Enzyme-linked immunosorbent assay}

Plasma HER2 $2_{\text {ECD }}$ was quantified by means of a commercially available ELISA (Siemens Healthcare Diagnostics, Inc., Tarrytown, NY, USA) according to the manufacturers' instructions. This is the same kit today approved by the FDA and available by Martell Diagnostic Laboratories, Roseville, MN, USA. Color intensity was measured on a COBAS EIA spectrophotometer (Hoffmann-La Roche Ltd., Basel, Switzerland). In general, samples were analyzed in batches every 6 months. Internal quality control was performed during each run using the controls provided with each kit. The results were expressed in nanogram per milliliter ( $\mathrm{ng} / \mathrm{ml}$ ). Each sample, standard, and control was assayed in duplicate. Inter- and intra-assay coefficients of variation were less than $10 \%$.

\section{Statistical analysis}

HER $2_{\text {ECD }}$ baseline levels just before and after trastuzumab infusion were compared to each other and to the 
Table 1 Clinicopathological patient's characteristics

\begin{tabular}{|c|c|c|}
\hline & $\begin{array}{l}\text { Arm A } \\
(n=66)\end{array}$ & $\begin{array}{l}\text { Arm B } \\
(n=65)\end{array}$ \\
\hline \multicolumn{3}{|l|}{ Age (years) } \\
\hline Median (range) & $54(33-78)$ & $57(33-72)$ \\
\hline \multicolumn{3}{|l|}{ ECOG PS } \\
\hline Unknown & $1(2 \%)$ & \\
\hline $0-1$ & 65 (99\%) & $65(100 \%)$ \\
\hline \multicolumn{3}{|l|}{$\mathrm{N}^{\circ} \mathrm{CT}$ regimens } \\
\hline 1st line & 47 (71\%) & $46(71 \%)$ \\
\hline 2nd-3rd line & $19(29 \%)$ & $19(29 \%)$ \\
\hline \multicolumn{3}{|l|}{ ER status } \\
\hline Positive & $43(65 \%)$ & $38(58 \%)$ \\
\hline Negative & $23(35 \%)$ & $27(42 \%)$ \\
\hline \multicolumn{3}{|l|}{ Adjuvant Anthracyclines } \\
\hline No & $39(59 \%)$ & $44(68 \%)$ \\
\hline Yes & 27 (41\%) & $21(32 \%)$ \\
\hline \multicolumn{3}{|l|}{ Endocrine therapy } \\
\hline No & $14(21 \%)$ & $16(24 \%)$ \\
\hline Adjuvant & 19 (29\%) & $24(37 \%)$ \\
\hline Palliative & $8(12 \%)$ & $8(12 \%)$ \\
\hline \multicolumn{3}{|l|}{ Disease sites } \\
\hline Bone only & $6(9 \%)$ & $10(15 \%)$ \\
\hline Visceral only & $20(30 \%)$ & $31(48 \%)$ \\
\hline Visceral + bone & $24(36 \%)$ & $12(18 \%)$ \\
\hline Advanced disease at diagnosis (pM1) & $21(32 \%)$ & $24(37 \%)$ \\
\hline
\end{tabular}

primary tumor IHC HER2 scores, HER2 gene copy numbers, as well as oestrogen receptor (ER), progesterone receptor (PR) status using the Jonckheere-Terpstra and Wilcoxon rank sum test.

The test cut-off value recommended by the manufacturer in serum of primary and metastatic patients is 15 $\mathrm{ng} / \mathrm{ml}[18-21]$. This threshold is used to discriminate HER2+ from HER2 negative patients and is known to be indicative for bad prognosis under chemotherapy alone. Therefore, we analyzed the impact of this threshold value with respect to prediction to therapy response. Uni- and multivariate Cox-regression as well as the multitest function (Torsten Hothorn (2017) maxstat: Maximally Selected Rank Statistics. R package version 0.7-25. https://CRAN.R-project.org/package=maxstat) were used to investigate the clinical value of HER $2_{\mathrm{ECD}}$ levels with respect to time to progression (TTP) on combined trastuzumab-chemotherapy (TTP-TChemo) and to overall survival (OS).

Statistical analysis was performed with Statistical Package Software R (Version 3.4.1, 2017-06-30) www.rproject.org). $P$ values $<0.05$ were considered significant.

\section{Results}

Expression and association of baseline HER2 $2_{\mathrm{ECD}}$ levels with other makers

The HER $2_{\text {ECD }}$ levels just before $(n=131)$ and after $(n=$ 113) trastuzumab infusion varied within the normal repeated measurements variance independently from their levels. The detected ranges varied from 5 to 1427 $\mathrm{ng} / \mathrm{ml}$ (median $26 \mathrm{ng} / \mathrm{ml}$; mean $81 \mathrm{ng} / \mathrm{ml}$ ) and 5-1543 $\mathrm{ng} / \mathrm{ml}$ (median $27 \mathrm{ng} / \mathrm{ml}$; mean $76 \mathrm{ng} / \mathrm{ml}$ ) in samples before and after the first trastuzumab infusion with a resulting Pearson correlation coefficient of 0.995 . This extremely high correlation is independent from the treatment arm (Arm A: $r=0.997$; Arm B: $r=0.993$ ). Subsequently, we considered the mean of these two detected values as baseline if both values $(n=113)$ were available. Otherwise, we took the single available value.

No difference was found between the baseline HER $2_{\mathrm{ECD}}$ levels of patients in Arm $\mathrm{A}$ and $\mathrm{B}$, reflecting the random assignment of patients in the two arms.

Since the FDA approved threshold was based on serum detection, we compared the obtained plasma HER $2_{\mathrm{ECD}}$ data with the ones analyzed in serum of patients with similar age and menopausal status entering another trial (SAKK23/04; Fig. 1). The median value of both plasma and serum cohorts was $16 \mathrm{ng} / \mathrm{ml}$, similar to the one reported by the literature for HER2+ BC (12 to $15 \mathrm{ng} / \mathrm{ml}$ ) [22]. However, $40 \%$ of the HER $2_{\mathrm{ECD}}$ plasma values were above $30 \mathrm{ng} / \mathrm{ml}$, which represents the maximal values detected in serum samples of the SAKK23/ 04 and literature.

These plasma baseline mean values correlated positively with the HER2 gene ratio $\left(\mathrm{r}_{\mathrm{s}}=0.39, P<0.001\right)$ and HER2 protein expression levels $\left(\mathrm{r}_{\mathrm{s}}=0.36, P<\right.$ $0.001)$. The HER $2_{\mathrm{ECD}}$ levels were found significantly $(P<0.001)$ higher in patients with HER2 Dako 3+ (median; range: $40 ; 6-1485 \mathrm{ng} / \mathrm{ml} ; n=83$ ) as compared to Dako $2+(13 ; 7-138 \mathrm{ng} / \mathrm{ml} ; n=29)$ as detected locally in primary tumors. No significant difference was found between the HER $2_{\mathrm{ECD}}$ baseline values and other available parameters such as ER and PR expression $(P=0.64$ and $P=0.68)$, bone disease $(P=0.40)$ or number of metastatic sites $(P=0.37)$. Only a positive trend was observed between HER $2_{\mathrm{ECD}}$ levels and the presence of visceral disease $(P=0.05)$.

\section{Association of baseline HER2 $2_{E C D}$ levels with therapy response and clinical outcome}

Considering the threshold of $15 \mathrm{ng} / \mathrm{ml}$, Kaplan-Maier curves and log-rank analyses of the overall population (i.e. Arm A and B) revealed no significant impact of baseline HER $2_{\mathrm{ECD}}$ plasma values on disease progression (TTP and TTP-TChemo) or OS (Fig. 2a-c).

However, univariate Cox-regression analysis revealed a significant $(P=0.009)$ association between the HER $2_{\mathrm{ECD}}$ 
1000

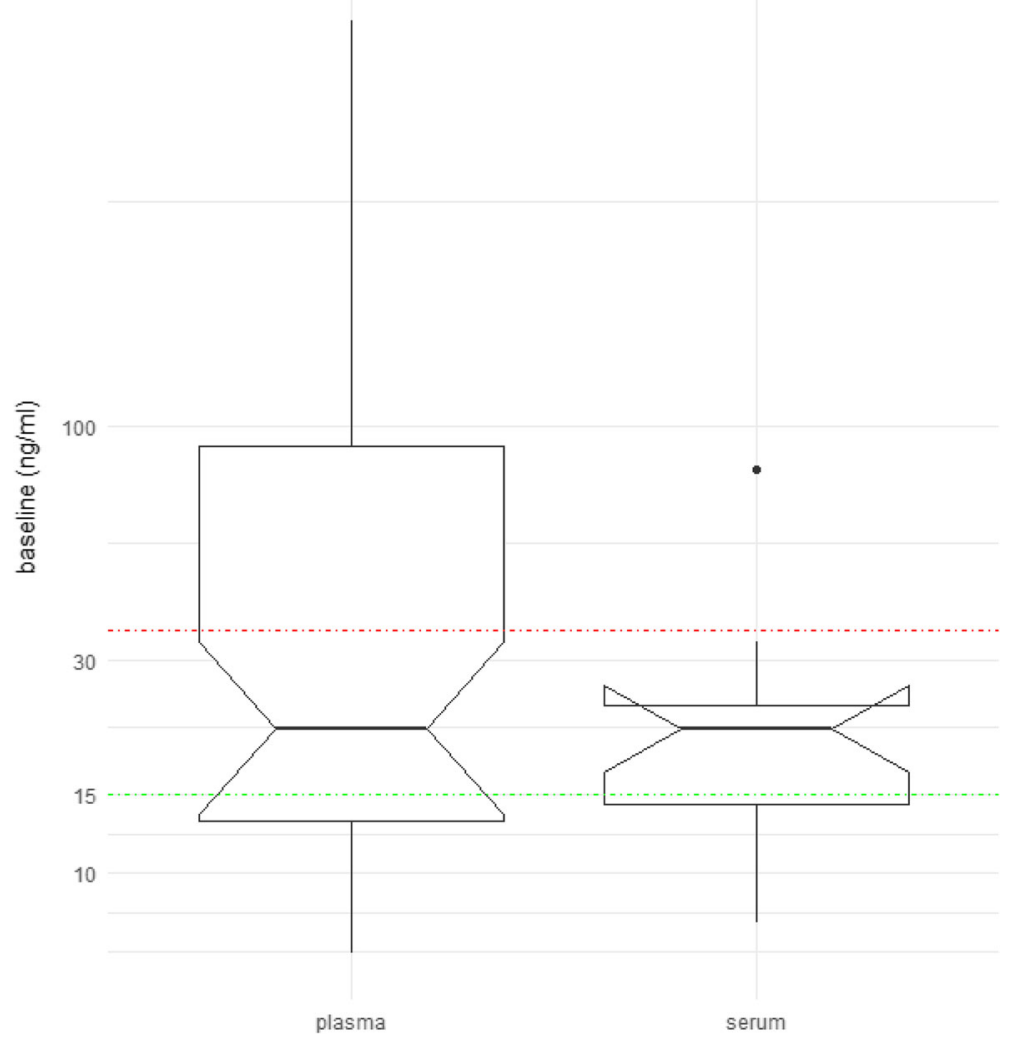

Fig. 1 Comparison of baseline ECDHER2 values as detected in plasma $(n=47)$ or serum $(n=13)$ in two SAKK studies. Median values of plasma ECDHER2 levels of selected postmenopausal patients with ER positive tumors (left) compared to the one detected in serum of patients entering the SAKK23/04 (in both studies median: $21 \mathrm{ng} / \mathrm{ml} ; P=0.42$ )

levels, when analyzed as a continuous variable, with respect to OS. Moreover, in a multivariate Hazard Coxregression analysis including baseline HER $2_{\mathrm{ECD}}$, ER- and PR-Status, treatment arm and the presence/absence of bone or visceral diseases, baseline HER $2_{\mathrm{ECD}}$ retained an independent significant prognostic factor (HR:1.2; CI: 1.1-1.3 using logarithmic transformed data; $P=0.026$ ) with respect to OS. In this model the presence of visceral disease was the only other significant parameter (HR:1.6; CI:1.3-2.2; $P=0.033$ ).

Therefore, by means of maxstat functions we searched for the most appropriate threshold plasma value in our cohort of patients with advanced BC. As depicted in Fig. $2 \mathrm{~d}$, patients with baseline values $\geq 35 \mathrm{ng} / \mathrm{ml}$ had a significant $(P=0.04)$ worse OS compared to those with lower levels. After 4 years only $21.4 \%$ (CI:13-37\%) of the patients with baseline HER $2_{\mathrm{ECD}}$ levels $\geq 35 \mathrm{ng} / \mathrm{ml}$ were still alive, while the OS rate was of $40 \%$ (CI:30-53\%) for patients with lower HER2 $2_{\mathrm{ECD}}$ levels.

Interestingly, patients in Arm A treated upfront with trastuzumab alone showed no OS difference regardless of the baseline HER2 $2_{\mathrm{ECD}}$ level (Fig. 3a), in contrast to the whole population (Fig. 2d). Conversely, the observed good prognostic HER2 $2_{\mathrm{ECD}}$ impact on OS is consistent for patients treated with TChemo upfront (Fig. 3b).

Of particular relevance, patients with low levels of baseline HER $2_{\mathrm{ECD}}(<15 \mathrm{ng} / \mathrm{ml})$ have a significantly better TTP-Tchemo if first treated with $\mathrm{T}$ alone. One year after study treatment start, no progression is observed in 58\% (CI:39-86\%) of patients treated in Arm A, compared to 28\% (CI:13-6\%) for patients in Arm B (Fig. 3c).

\section{Monitoring $\mathrm{HER} 2_{\mathrm{ECD}}$ levels during trastuzumab and TChemo treatments}

As shown in Fig. 4 and calculated by analysis of variance, we observed a significant time effect $(P=0.001)$ and time-treatment interaction $(P=0.0007)$. In case of therapy response, the HER $2_{\mathrm{ECD}}$ levels in patients receiving upfront TChemo (Arm B) decreased to a mean value of $15 \mathrm{ng} / \mathrm{ml}$. This decrease represents $>20 \%$ of the respective baseline values. These low values persisted until disease progression and rose thereafter. In contrast, HER2 $2_{E C D}$ levels did not change in patients responding at upfront trastuzumab alone (Arm A). Of interest, these values strongly increased (more than $20 \%$ of the respective baseline levels) at first disease progression (PD1). 
A

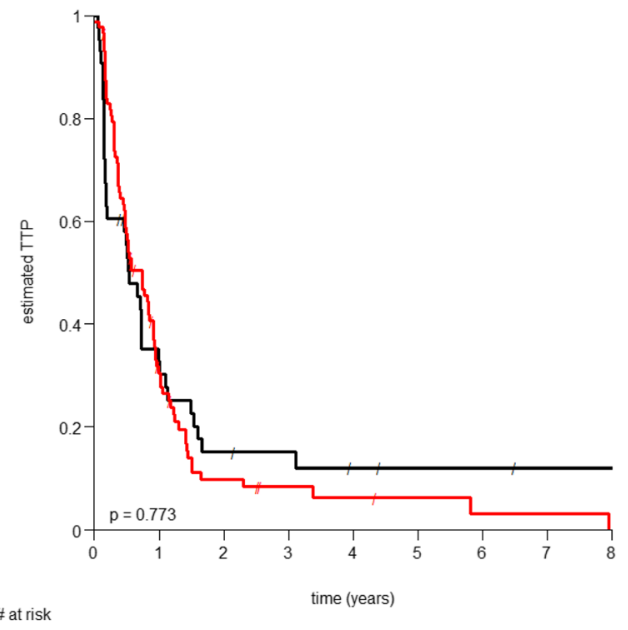

\# at risk
$<15 \mathrm{ng} / \mathrm{ml}$

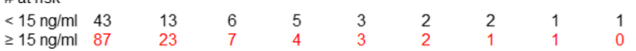

C

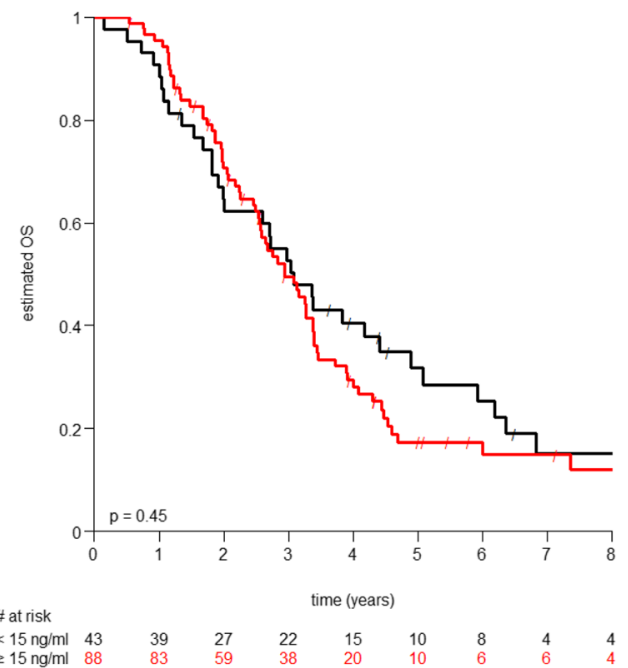

$B$

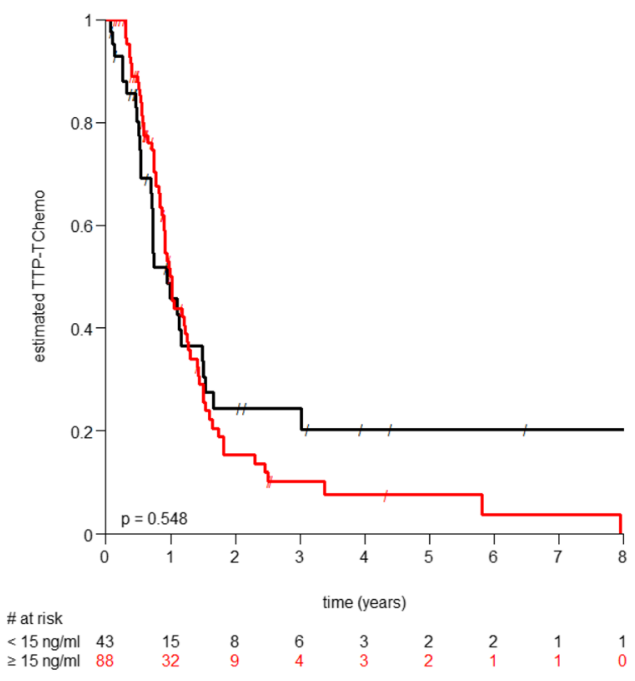

$D$

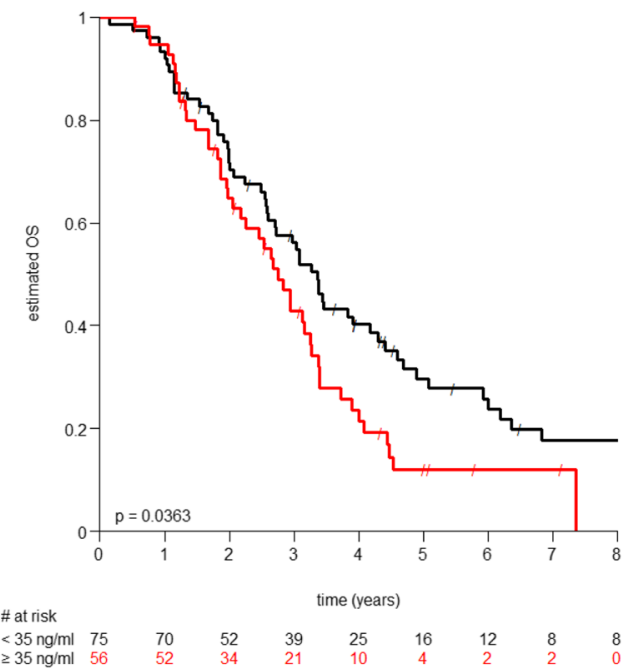

Fig. 2 Kaplan-Meyer curves of the overall study population with respect to a TTP, $\mathbf{b}$ TTP-TChemo; c OS using the threshold of $15 \mathrm{ng} / \mathrm{ml}$; and $\mathbf{d}$ OS using the calculated threshold of $35 \mathrm{ng} / \mathrm{ml}$

Lastly, we investigated if a decrease or increase within the first 9 weeks of at least $20 \%$ of HER $2_{\mathrm{ECD}}$ plasma levels with respect to the baseline values at first infusion could predict a longer TTP or OS. For patients treated upfront with trastuzumab alone (Arm A) there was a trend for better OS in case of quick decreased HER $2_{\mathrm{ECD}}$ levels versus worst OS in case of increased levels (Fig. 5).

\section{Discussion}

Our translational research study aimed to address the medical need to identify a robust non-invasive marker able to select patients with particular profit from a sequential anti-HER2 chemotherapy treatment strategy. An approach which, according to our results of the randomized Phase III trial SAKK22/99, might help deferring chemotherapy and its toxicity fitting in a de-escalating intention in the treatment of HER2-positive disease [17].

Our results indeed indicate that patients with low baseline HER $2_{\mathrm{ECD}}$ levels may profit of single agent trastuzumab, while no difference between arms is observed when the baseline HER2 $2_{\mathrm{ECD}}$ levels are higher (Fig. 3c/d). Moreover, since the HER $2_{\mathrm{ECD}}$ levels under trastuzumab 


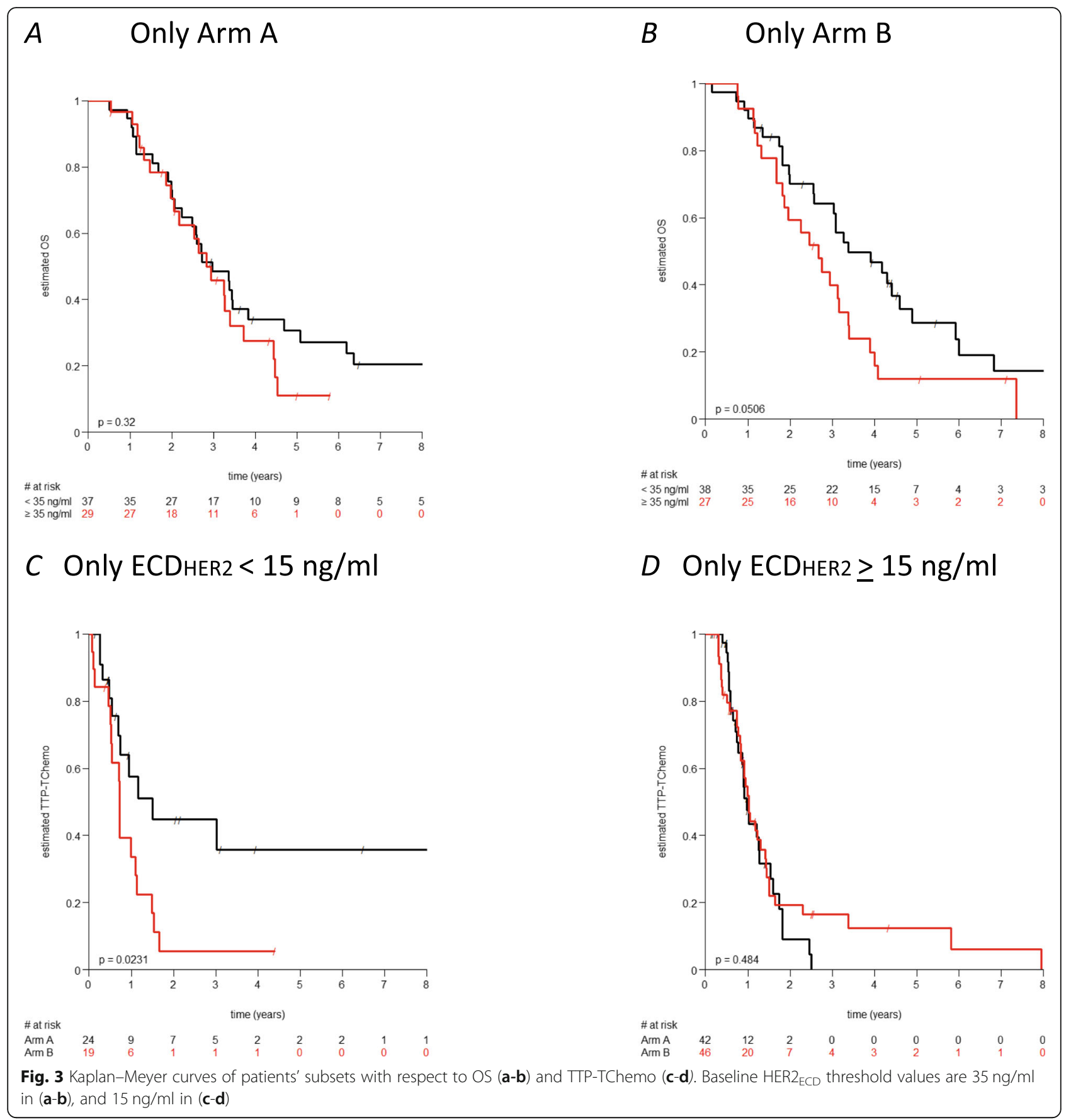

monotherapy remain fairly constant over time and start increasing only shortly before disease progression, monitoring HER $2_{\mathrm{ECD}}$ levels may help identifying the optimal moment for introducing chemotherapy.

To our best knowledge, this is the first study investigating plasma HER $2_{\mathrm{ECD}}$ or any other blood marker, including circulating tumor cells (CTCs) or free DNA, to address specifically the optimal time point for the introduction of chemotherapy after anti-HER2 monotherapy in HER2+ metastatic BC.
Several studies have investigated the value of serum HER $2_{\mathrm{ECD}}$ in patients with BC. Our results are in line with a recent review summarizing 27 studies (10 in metastatic BC, 13 in early $\mathrm{BC}$ and 4 in patients with early or metastatic BC) [18]. Even though there are discrepancies between studies, including the definition of the optimal threshold, the majority suggest that serum HER $2_{\text {ECD }}$ might be particularly useful in metastatic BC as an indicator of cancer progression and predictor for anti-HER2 therapies efficacy. 

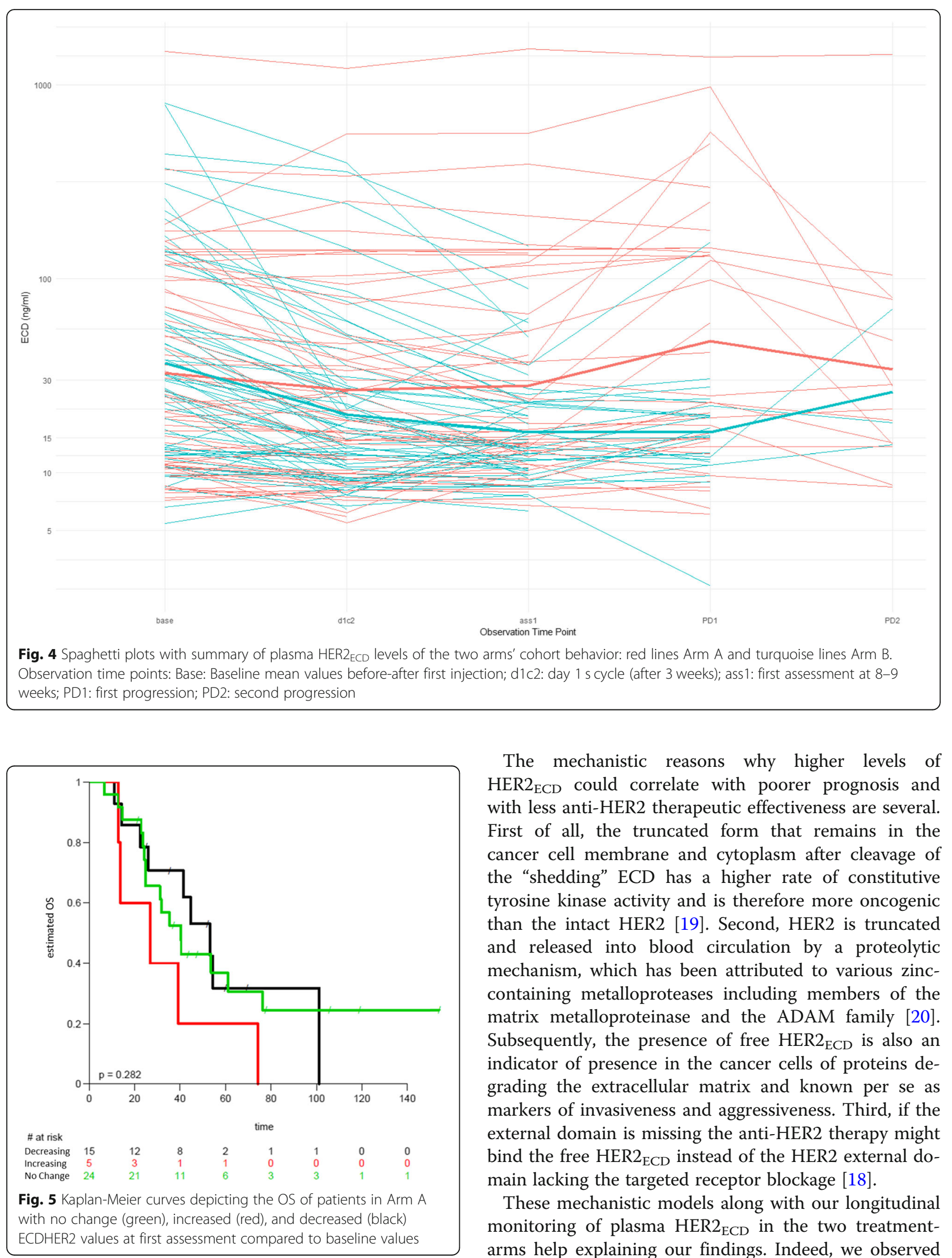

The mechanistic reasons why higher levels of HER $2_{\text {ECD }}$ could correlate with poorer prognosis and with less anti-HER2 therapeutic effectiveness are several. First of all, the truncated form that remains in the cancer cell membrane and cytoplasm after cleavage of the "shedding" ECD has a higher rate of constitutive tyrosine kinase activity and is therefore more oncogenic than the intact HER2 [19]. Second, HER2 is truncated and released into blood circulation by a proteolytic mechanism, which has been attributed to various zinccontaining metalloproteases including members of the matrix metalloproteinase and the ADAM family [20]. Subsequently, the presence of free HER $2_{\mathrm{ECD}}$ is also an indicator of presence in the cancer cells of proteins degrading the extracellular matrix and known per se as markers of invasiveness and aggressiveness. Third, if the external domain is missing the anti-HER2 therapy might bind the free HER $2_{\mathrm{ECD}}$ instead of the HER2 external domain lacking the targeted receptor blockage [18].

These mechanistic models along with our longitudinal monitoring of plasma HER $2_{\mathrm{ECD}}$ in the two treatmentarms help explaining our findings. Indeed, we observed 
longer TTP for patients with plasma HER2 $2_{\mathrm{ECD}}$ below 15 $\mathrm{ng} / \mathrm{ml}$ only when treated with sequential treatment. Trastuzumab alone in these patients binds to the fulllength HER2 domain and is effective. In this situation, there is no interaction between the free HER $2_{\mathrm{ECD}}$ and trastuzumab and the free HER $2_{\mathrm{ECD}}$ levels remain constant until the cancer progresses. At this point, the HER $2_{\mathrm{ECD}}$ levels increase and sequential HER $2_{\mathrm{ECD}}$ monitoring would allow identifying the exact moment for chemotherapy administration. According to protocol plasma collection timelines (baseline, after 3 weeks, at first response evaluation (8-9 weeks) and at PD) it was not possible to know how long before PD the HER $2_{\mathrm{ECD}}$ levels started to raise. Prospective studies should integrate monthly detections in order to estimate the possible anticipation of chemotherapy administration.

In contrast, we observed that HER $2_{\mathrm{ECD}}$ levels in patients treated with upfront TChemo, as reported in several other studies [21], continuously diminished, up to $<20 \%$ of the baseline level, when chemotherapy is effective. Consequently, patients with high levels of free ECD should be treated with upfront combination therapy.

Another recent meta-analysis based on 15 prospective and 8 retrospective studies, investigating the prognostic value of HER $2_{\mathrm{ECD}}$ with the FDA approved threshold of $15 \mathrm{ng} / \mathrm{ml}$, concluded that higher levels are correlated with poorer OS with a hazard ratio (HR) of 2.3 (CI:2.02.6) [23]. Our results are very much in line with this meta-analysis despite our threshold, with respect to OS for patients treated with upfront TChemo, had to be set at a higher level $(35 \mathrm{ng} / \mathrm{ml})$.

One possible explanation for our higher threshold and overall expression values of the plasma HER $2_{\mathrm{ECD}}$ could be that the plasma test also detects the extracellular domain of HER2 present on CTCs. If this hypothesis is correct, plasma HER $2_{\mathrm{ECD}}$ could be more sensitive and representative of the aggressiveness of the disease and subsequently more clinically useful than the serum test, indicating the concomitant presence of cleaving proteases and CTCs facilitating metastatic processes.

Further, in this translational study, we confirmed that monitoring of $\mathrm{HER} 2_{\mathrm{ECD}}$ values represent a clinically relevant complementary assessment in order to compensate inter-laboratory and inter-observer discrepancies in HER2 overexpression, particularly when detected in small biopsies of metastatic disease or in very old tissue specimens.

Another relevant finding is the high correlation of plasma levels at baseline and within $24 \mathrm{~h}$ after trastuzumab infusion in both arms. This observation is clinically important since it supplies evidence for avoiding unnecessary double blood assessments and suggests that trastuzumab does not bind immediately to the free soluble HER $2_{\mathrm{ECD}}$.

This study was well conceived at time of trial start almost 20 years ago. Unfortunately, the trial recruited too slowly and did not achieve the planned number of patients. Therefore, the overall analysis is underpowered and the current treatment of choice pertuzumab/trastuzumab is investigated in a more recent study (SAKK22/10).

\section{Conclusion}

In conclusion, our data suggest the detection of plasma HER $2_{\mathrm{ECD}}$ may help identifying patients with HER2+ BC who would profit from a sequential treatment and deciding when to introduce chemotherapy. According to our study and to the available literature serum or plasma HER $2_{E C D}$ levels should enter routine clinical practice for monitoring metastatic HER2+ BC. Even though finetuning on the threshold is necessary, serum and plasma HER2 ${ }_{\text {ECD }}$ might help to personalize anti-HER2 and chemotherapy regimens.

\section{Abbreviations}

BC: Breast cancer; CTC: Circulating tumor cells; ECD: Extracellular domain; ER: Estrogen receptor; FDA: Food \& Drug Administration; HER2: Epidermal growth factor receptor-2; HER2 $E C D$ : HER2 extracellular domain; HR: Hazard ratio; iv: intravenous; OS: Overall survival; PD: Progressive disease; PR: Progesterone receptor; SAKK: Swiss Group for Clinical Cancer Research; T: Trastuzumab; TChemo: Trastuzumab with chemotherapy; TKI: Tyrosine kinase inhibitor; TTP: Time to progression; TTP-TChemo: TTP on combined trastuzumab-chemotherapy

\section{Acknowledgements}

We thank patients, investigators, nurses and data managers of the 9 participating centers in Switzerland and trial coordinators of SAKK for their excellent support in recruiting the plasma samples. We are indebted to the former Stiftung Tumobank Basel (STB) particularly to Prof. Urs Eppenberger, Prof. Heinz Müller, Dr. Willy Küng and Dr. Vincent Vuaroqueaux for all type of support. Lastly, we are grateful to all technical personnel of the STB and at Pathology USB for the perfect performance of the ELISA test.

\section{Authors' contributions}

SE, OP, BT made substantial contributions to conception and design of the study. TR, OP, BT made substantial contributions to acquisition of samples. SE and DAR were responsible for samples analysis and logistic. DK, DD and SE analyzed the produced data. SE, DK, KR, TR, OP, BT were involved in drafting the manuscript or revising it critically for important intellectual content. All authors read and approved the final manuscript.

\section{Funding}

This work was supported by SAKK (Swiss Group for Clinical Cancer Research), that reimbursed in part the costs of the kits, the cost of sample collection at the centers and the salary of those performing statistical analyses and manuscript revising. The former Stiftung Tumorbank Basel (STB foundation) and afterword the Institute for medical genetics and pathology at the University Hospital of Basel supplied the infrastructure and personnel for logistics, test performance and sample storage. Study design and manuscript writing were scientific efforts of the researchers involved.

\section{Availability of data and materials}

All data are stored by the SAKK and rests of material is stored at the biobank of Pathology University hospital Basel and under the ownership of the SAKK. Data and rests of material are available from the SAKK on reasonable request. 


\section{Ethics approval and consent to participate}

Swissmedic Nr: 19995003229.

Swissmedic Authorisation date (IKS at that time): 18.08.1999.

Ethic committee Ticino for Istituto Oncologico della Svizzera Italian (IOSI) and Ospedale Regionale di Lugano Civico.

Kantonale Ethikkommission Bern for Inselspital and STS AG Thun, reference number 111/99.

Ethikkommission Nordwest- und Zentralschweiz (EKNZ) for Kantonsspital Aarau, Kantonsspital Baden, Universitätsspital Basel and Praxis Dr. K. Beretta, reference number M158/99.

Ethikkommission Ostschweiz (EKOS) for Kantonsspital St. Gallen.

Ethikkommission Zürich for Kantonsspital Graubünden, Stadtspital Triemli, Stadtspital Waid and Universitätsspital Zürich, reference number 595. Commission Cantonale d'Ethique de la Recherche sur l'être humain (CCER) for Hôpitaux Universitaires de Genève.

Commission cantonale d'éthique de la recherche sur l'être humain (CER-VD) for Centre hospitalier universtaire vaudois (CHUV), reference number F92/99. Comitato etico from the Istituto Europeo di Oncologia (IEO).

Comitato etico from the Ospedale di Circolo e Fondazione Macchi.

The study number received from some Ethical Committees is missed since at that time this did not apply.

All patients gave written inform consent and could disenroll from the study at any time. Indeed, we destroyed the blood samples of the two patients who retrieved their consent after blood donation.

\section{Consent for publication}

The SAKK board approved the publication of this manuscript.

\section{Competing interests}

Dirk Klingbiel performed the statistical analysis of the data working for the SAKK Swiss Group for Clinical Cancer Research Coordinating Center, Bern, Switzerland. Thereafter, before submission of the manuscript, he joined F. Hoffmann-La Roche Ltd. (F. Hoffmann-La Roche Ltd., Global Product Development Medical Affairs (Biometrics), CH-4053 Basel).

D.A. Rufle joined Ausbilder Biologie, AZM-2202.2.04, Lachmattstrasse 81, 4132 Muttenz after complete performance of all laboratory analyses.

B. Thürlimann holds stock of Roche and received honoraria for consultations from Roche.

All other authors declare that they have no competing interests.

\section{Author details}

${ }^{1}$ University Hospital Basel, Institute of Human Genetic and Pathology, Schönbeinstrasse 40, CH-4031 Basel, Switzerland. ${ }^{2}$ SAKK Swiss Group for Clinical Cancer Research Coordinating Centre, Bern, Switzerland. ${ }^{3} \mathrm{~F}$. Hoffmann-La Roche Ltd, Basel, Switzerland. "Brustzentrum Ostschweiz and University of Basel, St. Gallen, Switzerland. ${ }^{5}$ University of Basel, Basel, Switzerland. ${ }^{6}$ Breast Unit and Institute of Oncology of Southern Switzerland, Geneva University Hospitals, Swiss Group for Clinical Cancer Research (SAKK), Lugano, Viganello, Switzerland. 'Breast Centre St, Gallen, Switzerland.

\section{Received: 22 June 2019 Accepted: 31 January 2020}

Published online: 11 February 2020

\section{References}

1. Eppenberger-Castori S, Kueng W, Benz C, Caduff R, Varga Z, Bannwart F, Fink D, Dieterich $H$, Hohl M, Muller $H$, et al. Prognostic and predictive significance of ErbB-2 breast tumor levels measured by enzyme immunoassay. J Clin Oncol. 2001;19(3):645-56.

2. Eppenberger-Castori S, Moore DH Jr, Thor AD, Edgerton SM, Kueng W, Eppenberger U, Benz CC. Age-associated biomarker profiles of human breast cancer. Int J Biochem Cell Biol. 2002;34(11):1318-30.

3. Perou CM, Sorlie T, Eisen MB, van de Rijn M, Jeffrey SS, Rees CA, Pollack JR, Ross DT, Johnsen H, Akslen LA, et al. Molecular portraits of human breast tumours. Nature. 2000;406(6797):747-52.

4. Urban P, Vuaroqueaux V, Labuhn M, Delorenzi M, Wirapati P, Wight E, Senn HJ, Benz C, Eppenberger U, Eppenberger-Castori S. Increased expression of urokinase-type plasminogen activator mRNA determines adverse prognosis in ErbB2-positive primary breast cancer. J Clin Oncol. 2006;24(26):4245-53.

5. Burki TK. T-DM1 for residual, invasive, HER2-positive breast cancer. Lancet Oncol. 2019;20(1):e13.
6. Cardoso F, Costa A, Senkus E, Aapro M, Andre F, Barrios CH, Bergh J, Bhattacharyya G, Biganzoli L, Cardoso MJ, et al. 3rd ESO-ESMO international consensus guidelines for advanced breast Cancer (ABC 3). Breast. 2017:31:244-59.

7. Moasser MM. Targeting the function of the HER2 oncogene in human cancer therapeutics. Oncogene. 2007;26(46):6577-92.

8. Spector N. Treatment of metastatic ErbB2-positive breast cancer: options after progression on trastuzumab. Clin Breast Cancer. 2008;8(Suppl 3):S94-9.

9. Brandt-Rauf PW, Pincus MR, Carney WP. The c-erbB-2 protein in oncogenesis: molecular structure to molecular epidemiology. Crit Rev Oncog. 1994;5(2-3):313-29.

10. Ali SM, Leitzel K, Lipton A, Carney WP, Kostler WJ. Value of serum human epidermal growth factor receptor 2 (HER2)/neu testing for early prediction of response to HER2/neu-directed therapies is still an open one and deserves further study in large prospective trials. J Clin Oncol. 2009;27(36): e273 author reply e274-275.

11. Ali SM, Carney WP, Esteva FJ, Fornier M, Harris L, Kostler WJ, Lotz JP, Luftner D, Pichon MF, Lipton A, et al. Serum HER-2/neu and relative resistance to trastuzumab-based therapy in patients with metastatic breast cancer. Cancer. 2008;113(6):1294-301.

12. Brandt-Rauf PW, Luo JC, Carney WP, Smith S, De Vivo I, Milling C, Hemminki $\mathrm{K}$, Koskinen $\mathrm{H}$, Vainio $\mathrm{H}$, Neugut Al. Detection of increased amounts of the extracellular domain of the c-erbB-2 oncoprotein in serum during pulmonary carcinogenesis in humans. Int J Cancer. 1994;56(3):383-6.

13. Liedtke C, Broglio K, Moulder S, Hsu L, Kau SW, Symmans WF, Albarracin C, Meric-Bernstam F, Woodward W, Theriault RL, et al. Prognostic impact of discordance between triple-receptor measurements in primary and recurrent breast cancer. Ann Oncol. 2009;20(12):1953-8.

14. Solomayer EF, Becker S, Pergola-Becker G, Bachmann R, Kramer B, Vogel U, Neubauer H, Wallwiener D, Huober J, Fehm TN. Comparison of HER2 status between primary tumor and disseminated tumor cells in primary breast cancer patients. Breast Cancer Res Treat. 2006;98(2):179-84.

15. Chan A, Morey A, Brown B, Hastrich D, Willsher P, Ingram D. A retrospective study investigating the rate of HER2 discordance between primary breast carcinoma and locoregional or metastatic disease. BMC Cancer. 2012;12:555.

16. Carney WP, Bernhardt D, Jasani B. Circulating HER2 extracellular domain: a specific and quantitative biomarker of prognostic value in all breast Cancer patients? Biomark Cancer. 2013;5:31-9.

17. Pagani O, Klingbiel D, Ruhstaller T, Nole F, Eppenberger S, Oehlschlegel C, Bernhard J, Brauchli P, Hess D, Mamot C, et al. Do all patients with advanced HER2 positive breast cancer need upfront-chemo when receiving trastuzumab? Randomized phase III trial SAKK 22/99. Ann Oncol. 2017;28(2):305-12.

18. Perrier A, Gligorov J, Lefevre G, Boissan M. The extracellular domain of Her2 in serum as a biomarker of breast cancer. Lab Investig. 2018;98(6):696-707.

19. Christianson TA, Doherty JK, Lin YJ, Ramsey EE, Holmes R, Keenan EJ, Clinton GM. NH2-terminally truncated HER-2/neu protein: relationship with shedding of the extracellular domain and with prognostic factors in breast cancer. Cancer Res. 1998;58(22):5123-9.

20. Duffy MJ, McKiernan E, O'Donovan N, McGowan PM. Role of ADAMs in cancer formation and progression. Clin Cancer Res. 2009;15(4):1140-4.

21. Muller V, Witzel I, Luck HJ, Kohler G, von Minckwitz G, Mobus V, Sattler D, Wilczak W, Loning T, Janicke F, et al. Prognostic and predictive impact of the HER-2/ neu extracellular domain (ECD) in the serum of patients treated with chemotherapy for metastatic breast cancer. Breast Cancer Res Treat. 2004;86(1):9-18.

22. Witzel I, Loibl S, von Minckwitz G, Mundhenke C, Huober J, Hanusch C, Henschen S, Hauschild M, Lantzsch T, Tesch $\mathrm{H}$, et al. Monitoring serum HER2 levels during neoadjuvant trastuzumab treatment within the GeparQuattro trial. Breast Cancer Res Treat. 2010;123(2):437-45.

23. Zhang Z, Li C, Fan H, Xiang Q, Xu L, Liu Q, Zhou S, Xie Q, Chen S, Mu G, et al. Prognostic value of baseline serum HER2 extracellular domain level with a cut-off value of $15 \mathrm{ng} / \mathrm{mL}$ in patients with breast cancer: a systematic review and meta-analysis. Breast Cancer Res Treat. 2018;172(3):513-21.

\section{Publisher's Note}

Springer Nature remains neutral with regard to jurisdictional claims in published maps and institutional affiliations. 